\title{
Halftoning Over a Hexagonal Grid
}

\author{
Pierre-Marc Jodoin and Victor Ostromoukhov \\ Université de Montréal \\ Montréal, Canada
}

\begin{abstract}
In this contribution, we present an optimal halftoning algorithm that uniformly distributes pixels over a hexagonal grid. This method is based on a slightly modified error-diffusion approach presented at SIGGRAPH $2001 .{ }^{1}$ Our algorithm's parameters are optimized using a simplex downhill search method together with a blue noise based cost function. We thus present a mathematical basis needed to perform spectral and spatial calculations on a hexagonal grid. The proposed algorithm can be used in a wide variety of printing and visualization tasks. We introduce an application where our errordiffusion technique can be directly used to produce clustered screen cells.
\end{abstract}

Keywords: halftoning, error-diffusion, hexagonal grid, blue noise, Fourier transform.

\section{INTRODUCTION}

\subsection{Motivations}

Digital halftoning is a technique for rendering images with a wide dynamic range on devices having a limited number of available intensity levels. Driving color and grayscale printers is a typical application of digital halftoning. Most modern laser and inkjet printers possess a limited number of available output intensity levels, whereas the input signal may be considered as continuous-tone. In most applications, the input and output signals are sampled on a square or rectangular grid. It is for this reason that most research in digital halftoning has been focused on halftoning using square (or orthogonal) grids. Only a small amount of work has been done on hexagonal grids. ${ }^{2-4}$

Traditional digital halftoning using orthogonal grids has made tremendous progress during the last ten years. A number of algorithms have been proposed that considerably increase the visual quality of the produced images. ${ }^{1,5-7}$ At the same time, none of the algorithms mentioned above can be easily reused with satisfactory results on hexagonal grids.

In the present contribution, we will try to fill this gap. We introduce an error-diffusion algorithm with optimized coefficients that produces decent output on a hexagonal grid. We base our algorithm essentially on the work made by Ostromoukhov ${ }^{1}$ and reusing ideas derived from other contributions. ${ }^{5,6,8,9}$

The motivations for our work were two-fold:

- first, from a theoretical point of view, generalization of existing halftoning algorithms to a hexagonal grid will lead to a better understanding of the fundamental digital halftoning algorithm - which is far from obvious;

- second, physical devices with a hexagonal organization of visualization elements do exist (e.g. displays with hexagonal organization of RGB spots, inkjet printers with hexagonal organization of elementary ink drops). It may be more appropriate to drive such entities directly, without passing through the intermediate orthogonal structure. An example of such application will be presented in section 4 .

Further author information:

Pierre-Marc Jodoin's e-mail: jodoinp@iro.umontreal.ca

Victor Ostromoukhov's e-mail: ostrom@iro.umontreal.ca 

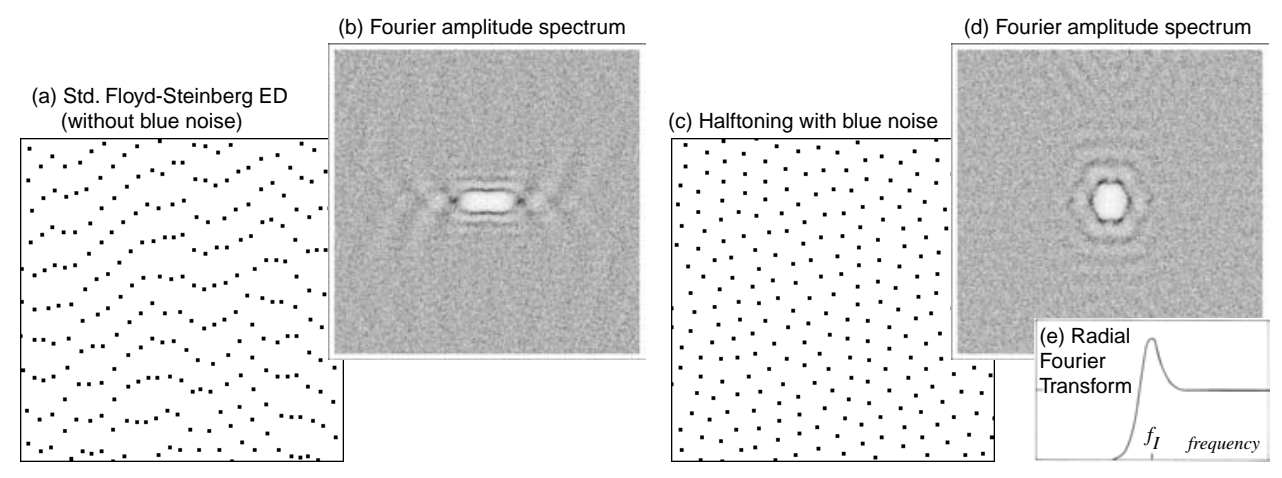

Figure 1. This figure illustrates the visual properties of blue noise: a light gray uniform patch halftoned with the scanline-path FloydSteinberg E-D algorithm (left) and with the algorithm proposed in ${ }^{1}$ (right). The Radial Fourier Transform (blue noise profile) was taken from Ulichney's book ${ }^{10}$.

\subsection{Error-Diffusion}

Error-diffusion is a widespread binarization algorithm that is mostly used to render images on black-and-white devices especially in the case where the preservation of fine image detail is needed. ${ }^{11}$ This algorithm is a fairly good compromise between speed, simplicity and visual quality.

The way error-diffusion works is simple. First, every input pixel $f(x, y)$ is selected one after the other. These pixels have a value ranging between 0 and 1 and the order in which they are processed depends on the chosen path (usually serpentine or scanline). When a pixel $f(x, y)$ is selected, it is compared to a fix threshold $t$ that is generally set at 0.5 . Whenever $f(x, y)$ is larger than $t$, the value " 1 " is assigned to the output image $g(x, y)$, otherwise it is set to " 0 ". This output value is then compared to the input intensity level and put their difference in the error image $e(x, y)$. The error is finally "distributed" to the neighboring pixels according to a given coefficient set similar to the one presented in Figure 3(a). The basic error-diffusion algorithm using a scanline path and the Floyd-Steinberg (FS) coefficient set is presented in Table 1.

Table 1. Classical error-diffusion algorithm processing the pixels in a scanline order using Floyd-Steinberg (FS) coefficient set.

\begin{tabular}{|c|c|}
\hline 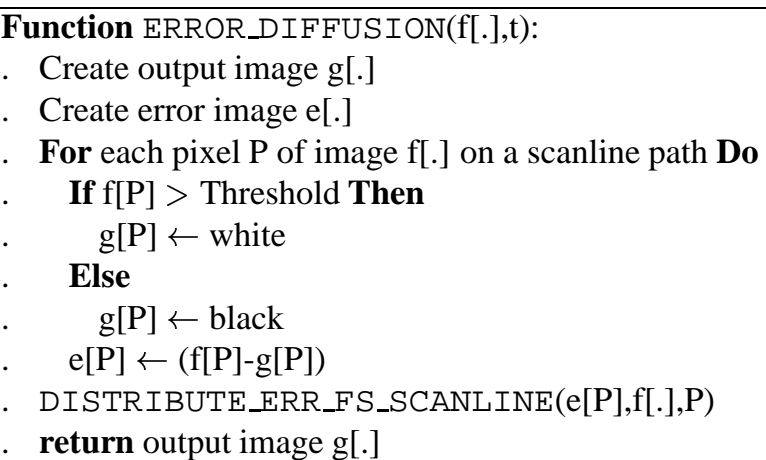 & $\begin{array}{l}\text { Function DISTRIBUTE_ERR_FS_SCANLINE }(E, \mathrm{f}[.], \mathrm{P}) \text { : } \\
\text {. } \mathrm{f}[\text { pixel at the right of } \mathrm{P}]+=E \times 7 / 16 \\
\text {. } \mathrm{f}[\text { pixel at the bottom right of } \mathrm{P}]+=E \times 1 / 16 \\
\text {. } \mathrm{f}[\text { pixel under } \mathrm{P}]+=E \times 5 / 16 \\
\text {. } \mathrm{f}[\text { pixel a the bottom left of } \mathrm{P}]+=E \times 3 / 16\end{array}$ \\
\hline
\end{tabular}

\subsection{Blue Noise}

Let us consider the problem of processing with a halftoning algorithm, an input image $f(x, y)$ of size $X_{s} \times Y_{s}$ where each pixel is assigned a constant intensity level $f(x, y)=I \in[0,1]$. The goal of any halftoning algorithm is to generate a bi-level output image $g(x, y)$ that approximates best the input image. The output must consequently contain $X{ }_{s} \times Y_{s} \times I$ 
Table 2. Pseudo-code used to calculate the radial Fourier transform (RFT) of a two dimensional Fourier amplitude spectrum (G[.]).

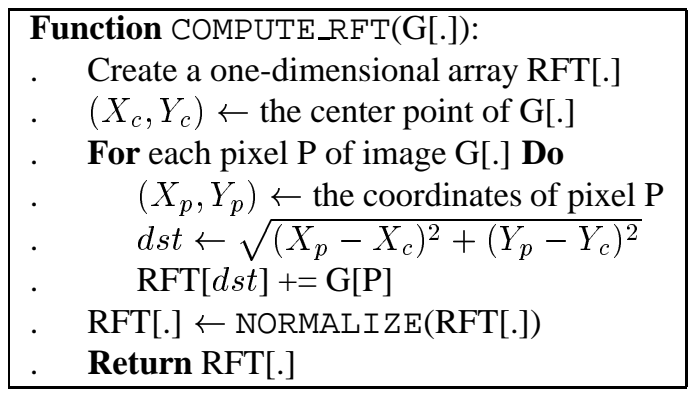

white pixels and $X_{s} \times Y_{s} \times(1-I)$ black pixels in order to have an average intensity level $I$. Each of the white pixels covers an area of $\frac{1}{I}$ pixel $^{2}$ and the average distance between each other is equivalent to

$$
\lambda_{I}=\left\{\begin{array}{cc}
\frac{1}{\sqrt{I}}, & \text { if } I \leq 0.5 \\
\frac{1}{\sqrt{1-I}}, & \text { otherwise. }
\end{array}\right.
$$

Since these pixels are assumed to be uniformly distributed, $\lambda_{I}$ induces a principal frequency $f_{I}=1 / \lambda_{I}{ }^{12,13}$ clearly visible in the frequency domain (see Figure 1 (d) and (e)). This kind of frequency shape with a peak over the principal frequency and radial symmetry is called the blue noise shape. As presented in Figure 1(e), the blue noise spectrum is often plotted as a one-dimensional profile function, often referred to as Radial Fourier Transform(RFT). The algorithm that computes RFT from $G(\alpha, \beta)$, the Fourier transform of the bi-level output image $g(x, y)$, is given by the pseudo-code in Table 2.

For the remainder of this article, after the problem statement in the next section, we will discuss the proposed algorithm along with its mathematical background and the optimization process in section 3. Section 4 will propose some possible applications for our algorithm while section 5 will comment on some results and section 6 will draw conclusions.

\section{PROBLEM STATEMENT}

The method presented in this paper was built upon the generic concept of a lattice. We define a lattice as being a twodimensional array of discrete points distributed over a coordinate system $\left(\vec{v}_{1}, \vec{v}_{2}\right)$ where $\vec{v}_{1}$ and $\vec{v}_{2}$ are unitary base vectors separated by a non-zero angle $\theta$. In this contribution, we call these points lattice points. By their very nature, these points cover no surface area and are labeled by a coordinate pair $(u, v)$. The Cartesian coordinates of a lattice point defined by its integer indices $u$ and $v$ are given by

$$
\Gamma(u, v)=u \vec{v}_{1}+v \vec{v}_{2}
$$

where $\Gamma($.$) is the conversion function. By using the well known Delaunay triangulation algorithm, we can subdivide$ the lattice into a set of triangles and find its dual structure called the Voronoi diagram. ${ }^{14}$ From the basic theory of computational geometry, we know that whenever all Delaunay triangles are equilateral, as in our case, the Voronoi regions are nothing other than perfect hexagonal cells. In the context of this paper, we give these cells the name hexagonal pixels. An example of this Voronoi diagram is presented in Figure 2.

Let us now consider a continuous input function $h(i, j)$ defined in the two-dimensional space $R^{2}$ where $h(i, j) \in R$. This function can be sampled over a lattice in such a way that each lattice point is associated with the function value at that point:

$$
f(u, v)=h(\Gamma(u, v))
$$

where $f(u, v)$ is the sampled function. 


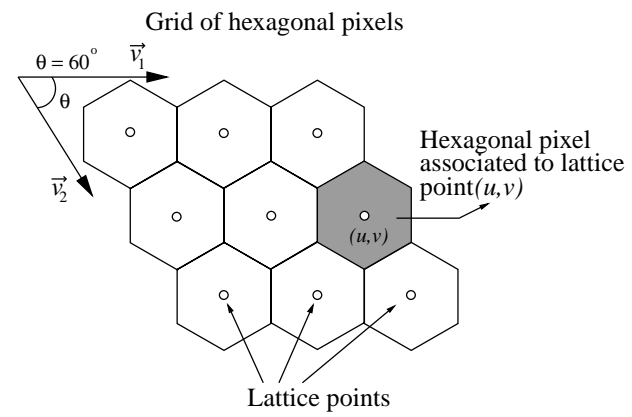

Figure 2. View of a hexagonal grid. Such a structure is a particular Voronoi diagram since each Voronoi region is a perfect hexagonal pixel.

It is well known in the halftoning community that a sampled function such as $f(u, v)$ can be approximated by a bi-level function $g_{h}(u, v)$. More precisely, a halftoning method refers to the process of representing a sampled function such as $f(u, v)$ over a limited number of values, typically set to 0 and 1 . The problem we solve in this contribution can therefore be formulated this way: from a two-dimensional function $f(u, v)$ sampled over a hexagonal lattice, generate a bi-level function $g_{h}(u, v)$ that best approximates $f(u, v)$. We consider that $g_{h}(u, v)$ approximates $f(u, v)$ well, whenever the following two requirements are respected:

1. The local intensity of $g_{h}(u, v)$ integrated over a small region equals $f(u, v)$. In other words,

$$
f(u, v)=\int_{u-\epsilon}^{u+\epsilon} \int_{v-\epsilon}^{v+\epsilon} g_{h}(u, v) d u d v
$$

where $\epsilon$ is a constant as large as a few hexagonal pixels.

2. If $f(u, v)=C$ for all values of $u$ and $v$ where $C$ is a constant, the binary pixels of $g_{h}(u, v)$ must be homogeneously distributed in space. In other words, $g_{h}(u, v)$ should have spectral blue noise characteristics as defined above.

To reach these requirements, we decided to use a modified version of the error-diffusion halftoning algorithm previously introduced.
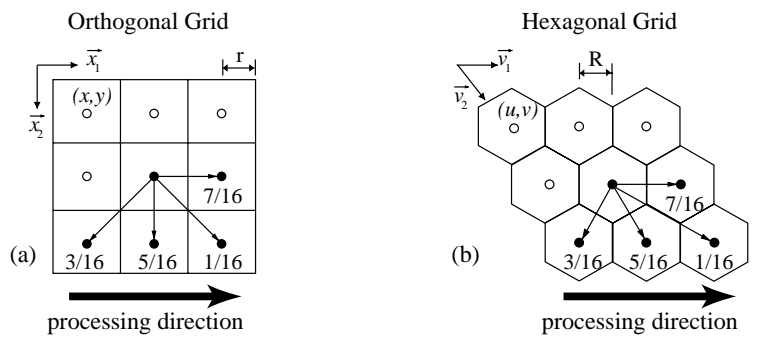

Figure 3. Example of the Floyd-Steinberg algorithm applied to an orthogonal grid (a) and to a hexagonal grid (b). The affine transformation between the orthogonal grid and the hexagonal grid is made by the bijection operator $\tau($.$) .$

\section{PROPOSED ALGORITHM}

\subsection{Model Description}

As we stated previously, with the help of an error-diffusion algorithm, our goal is to compute a halftone image $g{ }_{h}(u, v)$ that approximates a function $f(u, v)$ sampled over a hexagonal grid. Unfortunately, the basic error-diffusion algorithm 
presented in section 1.2 was not designed to work over hexagonal grids and thus generates disturbing artifacts. To solve that problem, we use a variable-coefficient approach ${ }^{1,9}$ which proposes to use 256 coefficient sets, one for each of the 256 intensity levels. Furthermore, the serpentine path was adopted to reduce directional artifacts mainly visible in the shadows and the highlights. ${ }^{15}$ The complete pseudo-code for this method using the notation of section 1.2 can be found in Table 3. Note that the 256 coefficient sets used in function DISTRIBUTE ERROR ( . ) will be further optimized by minimizing a given cost function built upon the blue noise constraint in order to meet section 2's requirements. That cost function will be described in detail in section 3.2.

Table 3. Pseudo-code of the error-diffusion algorithm used to distribute pixels over a hexagonal grid. Note that there is one coefficient set for each of the 256 intensity levels. Since the pixel value $f[P]$ was sampled between 0 and 1 , its multiplication by 255 gives an index corresponding to the appropriate coefficient set in the "Coeffs[.]" array.

\begin{tabular}{|c|c|}
\hline 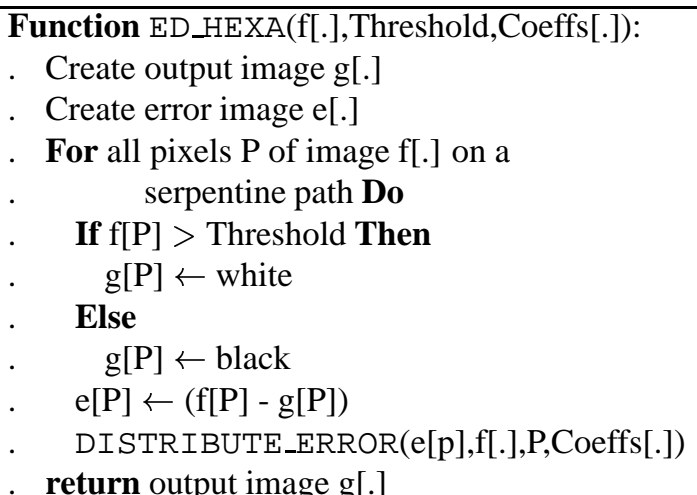 & 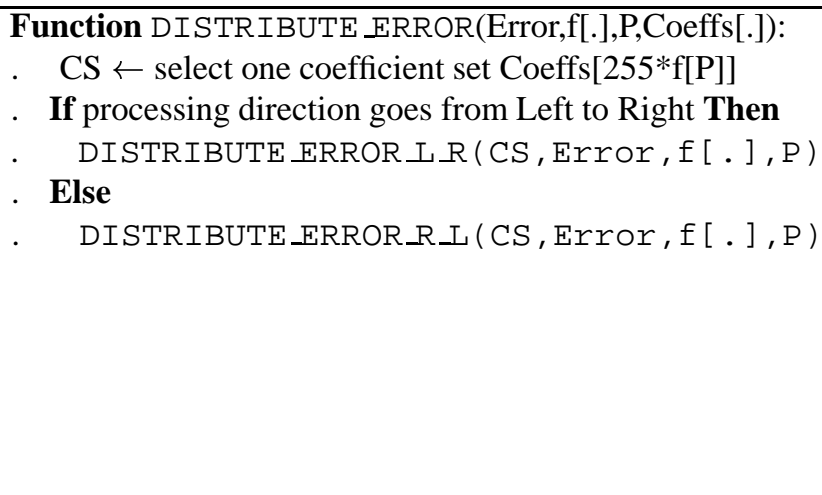 \\
\hline
\end{tabular}
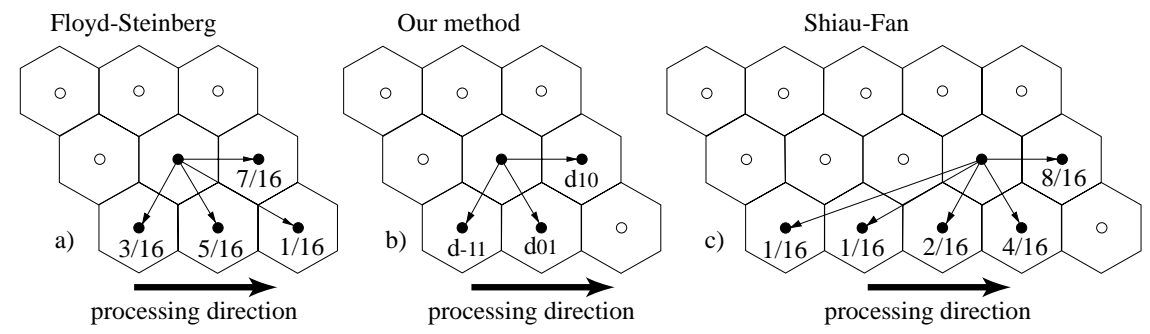

Figure 4. This figure shows how the error is distributed over the hexagonal pixels with Floyd-Steinberg's method, our method and Shiau-Fan's method.

\subsection{Optimization Process}

In the previous section, an error-diffusion algorithm (ED HEXA (. ) ) was proposed to uniformly distribute pixels on a hexagonal grid. However, in order to reach the blue noise requirement presented in section 2, we need to optimize the 256 coefficient sets. To do so, we implemented a simplex downhill method ${ }^{16}$ in combination with a blue noise based cost function and made it converge toward optimal coefficient values. This kind of method to find optimal coefficient weights has been used several times in the past. ${ }^{1,6,17}$ As shown in Figure 5, the function COST FUNCTION( $I$,set) computes the area between $G_{h}(\alpha, \beta)$ 's radial Fourier transform $R F T(f)$ and a given analytical function $F(f)$. It was found that for $F(f)$, a normalized Gaussian function with standard deviation $\sigma=\frac{f_{g}}{10}$ was a good compromise between simplicity and result quality. ${ }^{17}$ The cost function algorithm is presented in Table 4.

As suggested by Ostromoukhov, ${ }^{1}$ we did not optimise every 256 coefficient sets. To preserve stability of the whole optimization process, we only optimized a few key levels and linearly interpolated the coefficients between these specified 


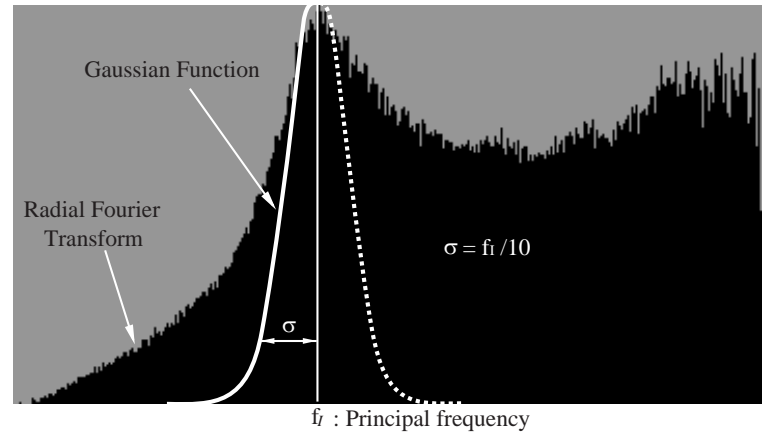

Figure 5. To calculate its error value, the function COST_FUNCTION $\left(I\right.$, set) first computes the hexagonal halftone image $g_{h}(u, v)$ of average intensity "I". It then computes a radial Fourier transform $R F T(f)$ and calculates a Gaussian function $F(f)$ centered on principal frequency $f_{I}{ }^{8,12} F(f)$ 's standard deviation is $\sigma=\frac{f_{I}}{10}$ and the cost is the area between $F(f)$ and $R F T(f): \operatorname{cost}=$ $\int_{f=0}^{f_{I}}\|R F T(f)-F(f)\| d f$

Table 4. Cost function used within the simplex downhill search method to find optimal coefficient sets

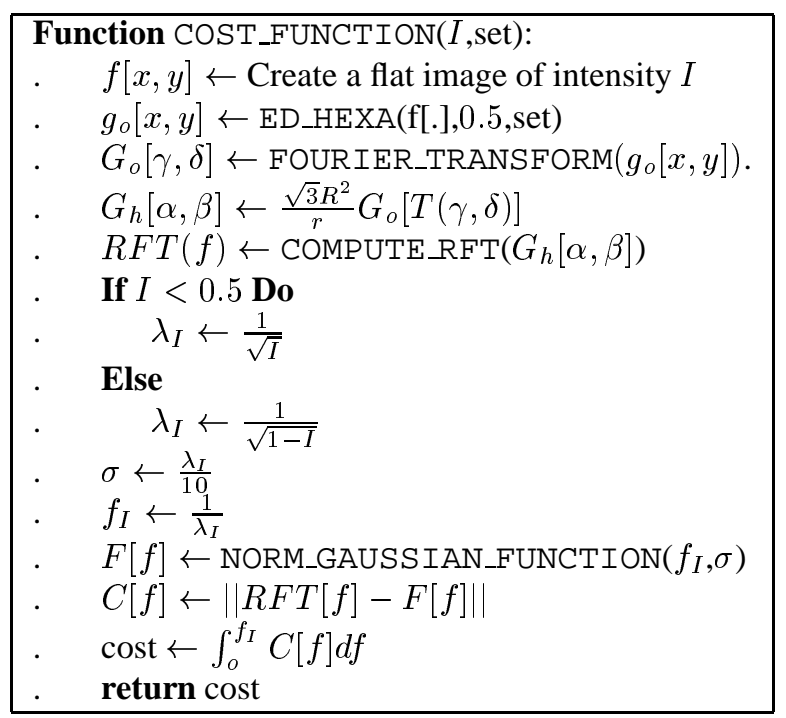

levels. Furthermore, because of error-diffusion's symmetry propriety above and under 0.5, we only optimized the first 128 coefficents and transposed it to the 128 others. The 128 optimized coefficient sets are presented in Table 6.

One aspect of the problem that was not tackled yet concerns the number of coefficients the coefficient sets must have. After trying many different configurations, we realized that the simple case with 3 coefficients $\left(d_{10}, d_{01}\right.$ and $d_{-11}$ as shown in Figure 4(b)) produces fairly good results. Furthermore, in contrast with the cases where more than 3 coefficients are used, the optimization process converges more rapidly and is less prone to fall into local minimas.

\subsection{Mathematical Context}

As presented in Figure 3(a), the error-diffusion algorithm was originally made to work over an orthogonal lattice. Also, it is more convenient to implement an orthogonal lattice in a computer program than a hexagonal lattice. For these reasons, we decided not to use ED_HEXA ( . ) during the optimization process to create the hexagonal halftone result $g_{h}(u, v)$. Instead, we used this algorithm on an orthogonal function $f_{o}(x, y)$ to create a bi-level output $g_{o}(x, y)$ also represented over an orthogonal grid. Once this last result is computed, its orthogonal lattice points are projected over 


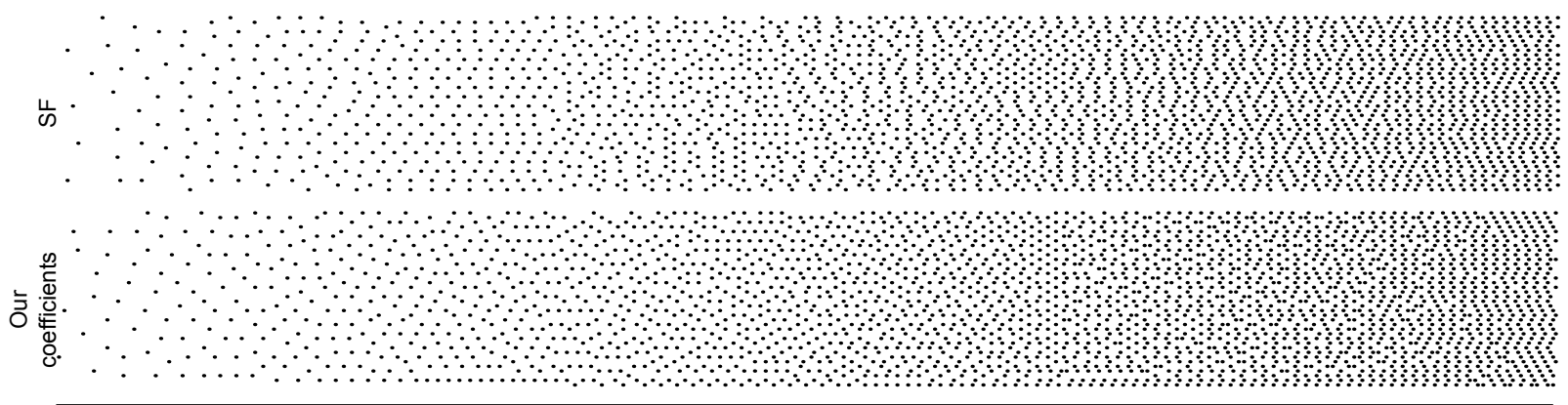

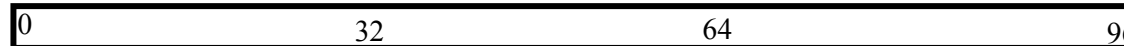

96
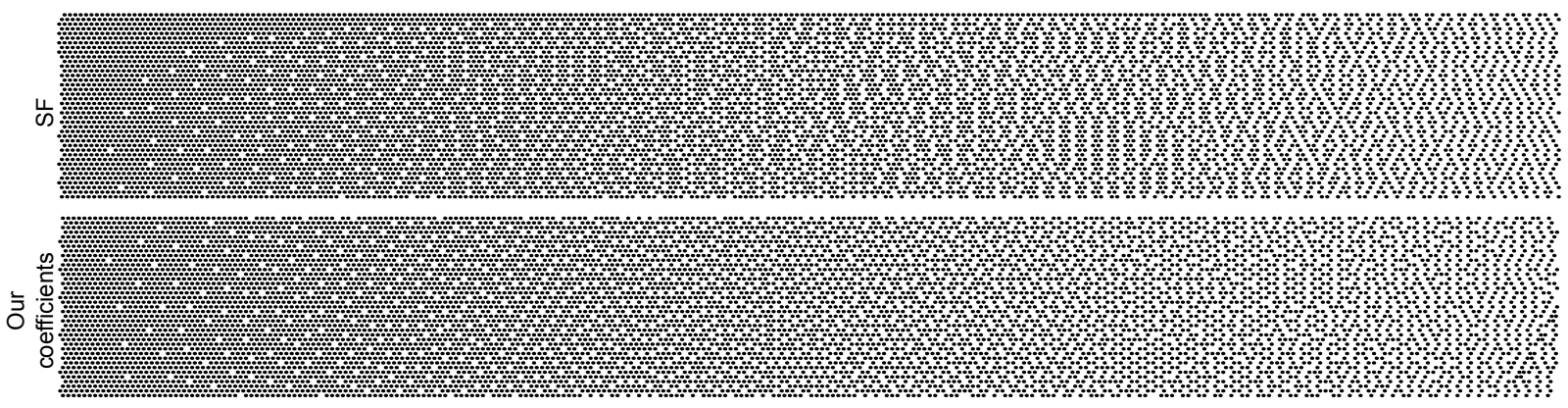

\begin{tabular}{|lllll}
\hline 256 & 224 & 192 & 160 & 128 \\
\hline
\end{tabular}

Figure 6. This figure represents a gray scale ramp sampled over a hexagonal lattice where each black dot stands for a lattice point. On top is the result of the ERROR_DIFFUSION (.) algorithm using a serpentine path and the well know Shiau-Fan(SF) coefficient set. ${ }^{5}$ At the bottom is the result of the ED_HEXA ( . ) algorithm using our optimized coefficients (see Table 6). There is a significant difference between these two approaches especially around levels 64, 96 160, 192 and 224

$g_{h}(u, v)$ 's hexagonal lattice points using a bijection operator $\tau($.$) (see Figure 3$ and 7). This operator has the general form $\tau(x, y)=(u, v)$. By using the coordinates of Figure 3(a) and (b), we find that for a given point $(x, y)$ defined on an orthonormal basis, its projection to a hexagonal basis is:

$$
\tau(x, y)=\left(\frac{R}{r} x+R y, \sqrt{3} R y\right)
$$

where $R$ is the hexagonal pixel half-size and $r$ is the orthogonal pixel half-size as shown in Figure 3. Images $g_{o}(x, y)$ and $g_{h}(u, v)$ can be linked together by equation (5) in order to get

$$
g_{h}(\tau(x, y))=g_{o}(x, y)
$$

In summary, during the optimization process, the error-diffusion method creates $g_{o}(x, y)$ and the bijection operator $\tau\left(\right.$.) maps this orthogonal grid to $g_{h}(u, v)$. To objectively optimize the method's parameters, we compute $g_{h}(u, v)$ 's Fourier transform $\left(G_{h}(\alpha, \beta)\right)$ and see how close it is to the blue noise shape. For a matter of convenience, even if $G_{h}(\alpha, \beta)$ could be computed by directly applying the Fourier transform to $g_{h}(u, v)$, we made the decision to convert the affine transformation $\tau($.$) to the Fourier domain. As shown in { }^{18}$, the spatial affine transformation of equation (6) can be directly transposed to the frequency domain as follows:

$$
G_{h}\left(\frac{r}{R} \gamma, \frac{\delta-\gamma r}{\sqrt{3} R}\right)=\frac{\sqrt{3} R^{2}}{r} G_{o}(\gamma, \delta)
$$

where $G_{o}(\gamma, \delta)$ is the Fourier transform of $g_{o}(x, y)$. The spectral bijection $\mathbf{T}($.$) can thus be represented by the following$ 
equation:

$$
\mathbf{T}(\gamma, \delta)=\left(\frac{r}{R} \gamma, \frac{\delta-\gamma r}{\sqrt{3} R}\right)
$$

The relation between the two bijection operators $\tau$ and $\mathbf{T}$ is illustrated in Figure 7.

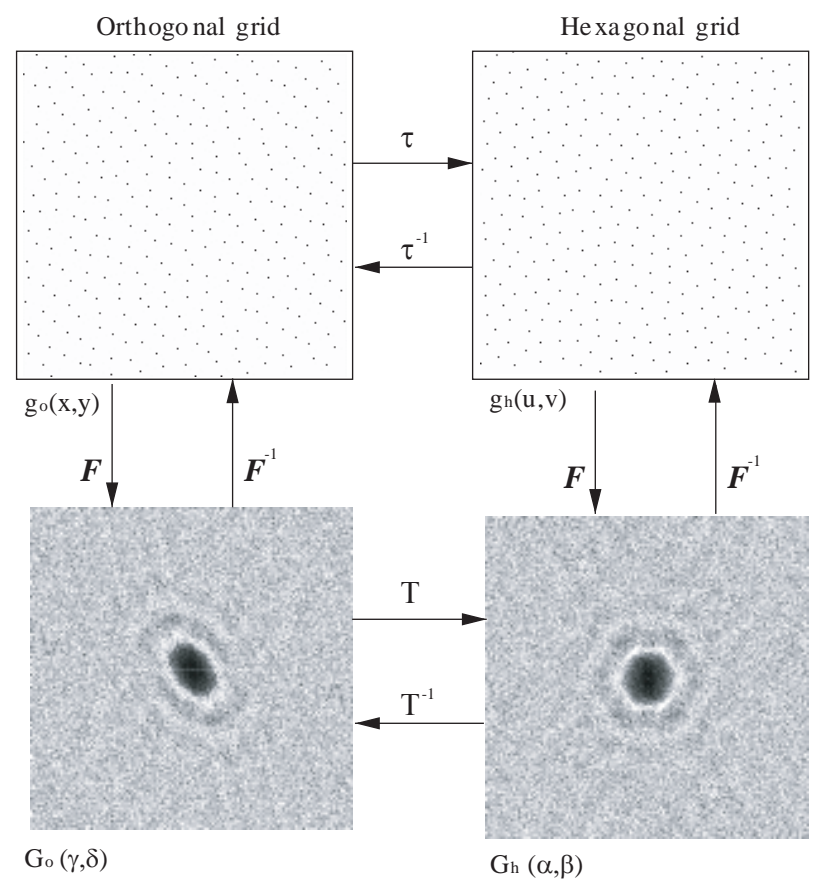

Figure 7. The following illustrates the process pipeline where the affine bijection $\tau\left(\right.$.) converts the orthogonal image $g_{o}(x, y)$ into the hexagonal one $g_{h}(u, v)$ and the spectral bijection $\mathbf{T}($.$) converts the Fourier transform G_{o}(\gamma, \delta)$ into $G_{h}(\alpha, \beta)$. $\mathbf{F}($.$) is the Fourier$ transform function.

\section{POSSIBLE APPLICATIONS}

By its very nature, our method preserves small image details better than most concurrent approaches. ${ }^{19}$ However, even if such a method is known for generating some of the most pleasing visual results, it is not suitable for a large variety of high resolution laser printers. ${ }^{19,20}$ Mostly because of the dot gain effect, the individual dots dispersed over the output image by the error-diffusion algorithm are hardly printable. For that reason, laser printers are driven most of the time by clustered-dot halftone methods. For example, to work around the dot gain effect, many printer drivers take advantage of a threshold matrix with a clustered organization of its dots, hence the generic name clustered-dot for this kind of matrix (see Figure 8(a)). ${ }^{15}$ Even if such a threshold algorithm is at the same time, fast, easy to implement and well suited for most laser printing devices, it experiences some serious limitations. First, in low resolution, it generates poor results since it filters all high frequency details. ${ }^{19}$ Second, it can only generates a fix number of gray levels which can produce visible steps in some cases. For example, the matrix shown in Figure 8(a) can only generate 12 different intensity levels and thus induces unacceptable false contours such as those in Figure 10(a).

One solution to these limitations, is to join together the clustered-dot algorithm with the error-diffusion method. That way, we can expect that the advantages of one will minimize the drawbacks of the other. Even if similar ideas were already proposed in the past, ${ }^{2,3,21}$ none of these approaches offers optimal coefficient sets. In this contribution, we apply our ED_HEXA ( . ) algorithm (in addition with the optimized coefficient sets) over a quasi-hexagonal grid such as the one in Figure 8(b). As Fan proposed it in ${ }^{3}$, instead of processing the individual pixels with the error-diffusion method, we 
process the individual threshold clusters. We thus sequentially compute the local error cumulated over each cluster and redistribute it to its neighbors (see Figure 8(b)). To do so, we slightly modified the ED HEXA (.) procedure to fit the quasi-hexagonal grid and called the new version ED _QUASI HEXA ( . ) as presented in Table 5.

Table 5. Modified error-diffusion algorithm used over a quasi-hexagonal grid such as the one in Figure 8(b).

\begin{tabular}{|c|c|}
\hline 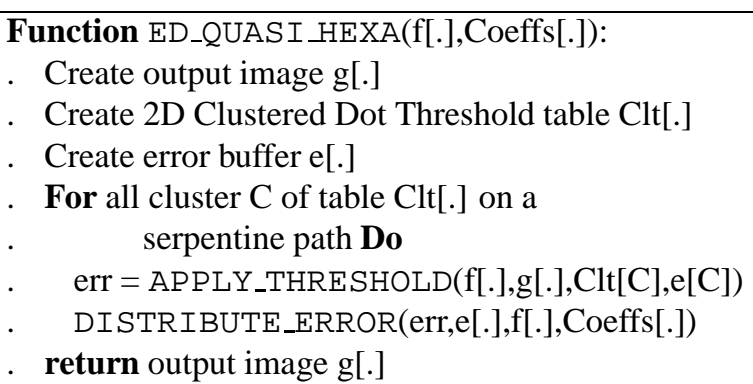 & 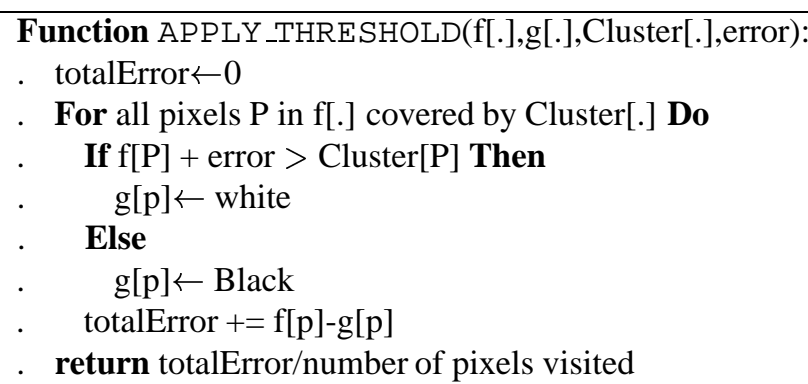 \\
\hline
\end{tabular}

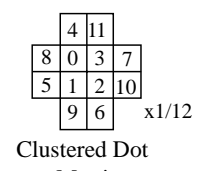

(a) Matrix

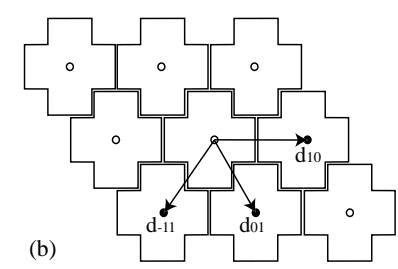

Figure 8. (a) A 12 level Clustered-dot threshold matrix and (b) the way error is distributed over a quasi-hexagonal grid.

\section{RESULTS}

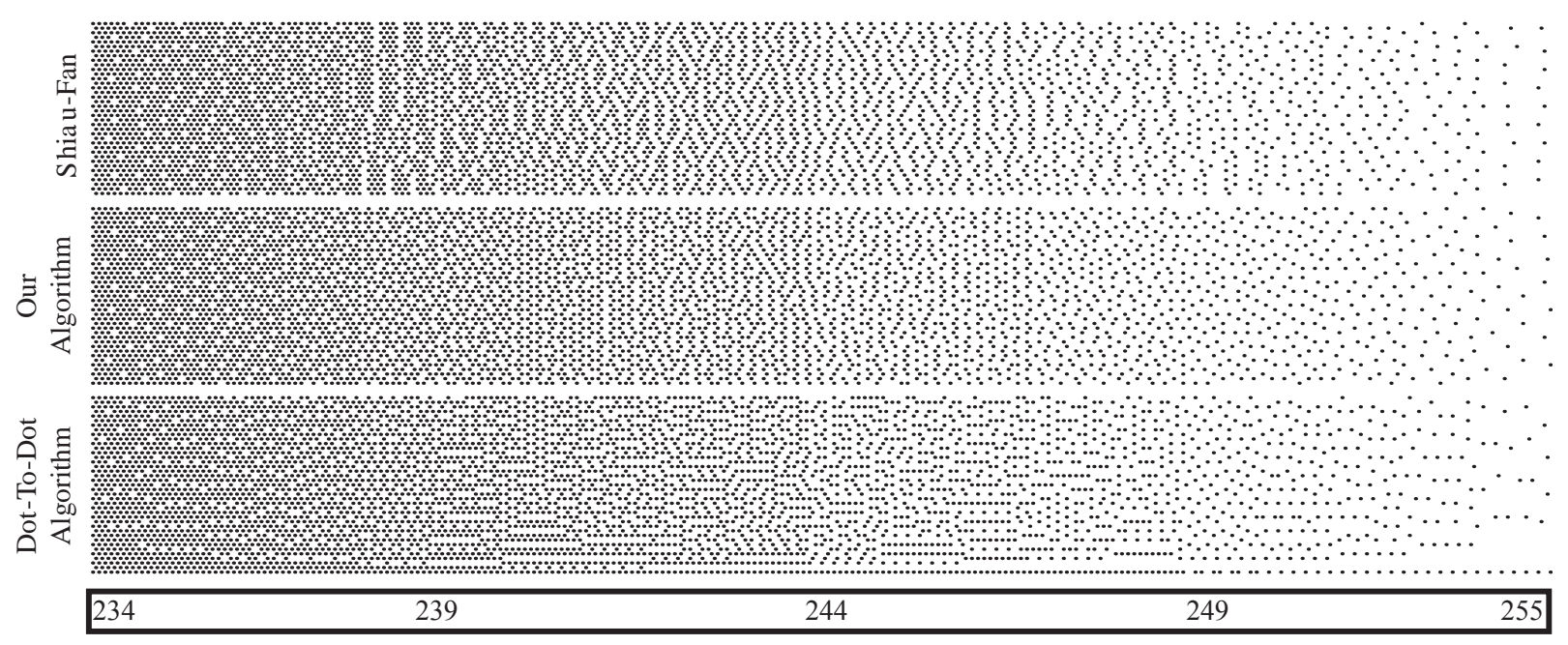

Figure 9. Result of 3 different methods applied on a quasi-hexagonal grid.

The final algorithm is fast, conceptually simple and memory efficient since it requires only a few operations per pixel. All computations needed for optimization of the ED coefficients are done once. These optimized coefficients are hardcoded into the final algorithm. 

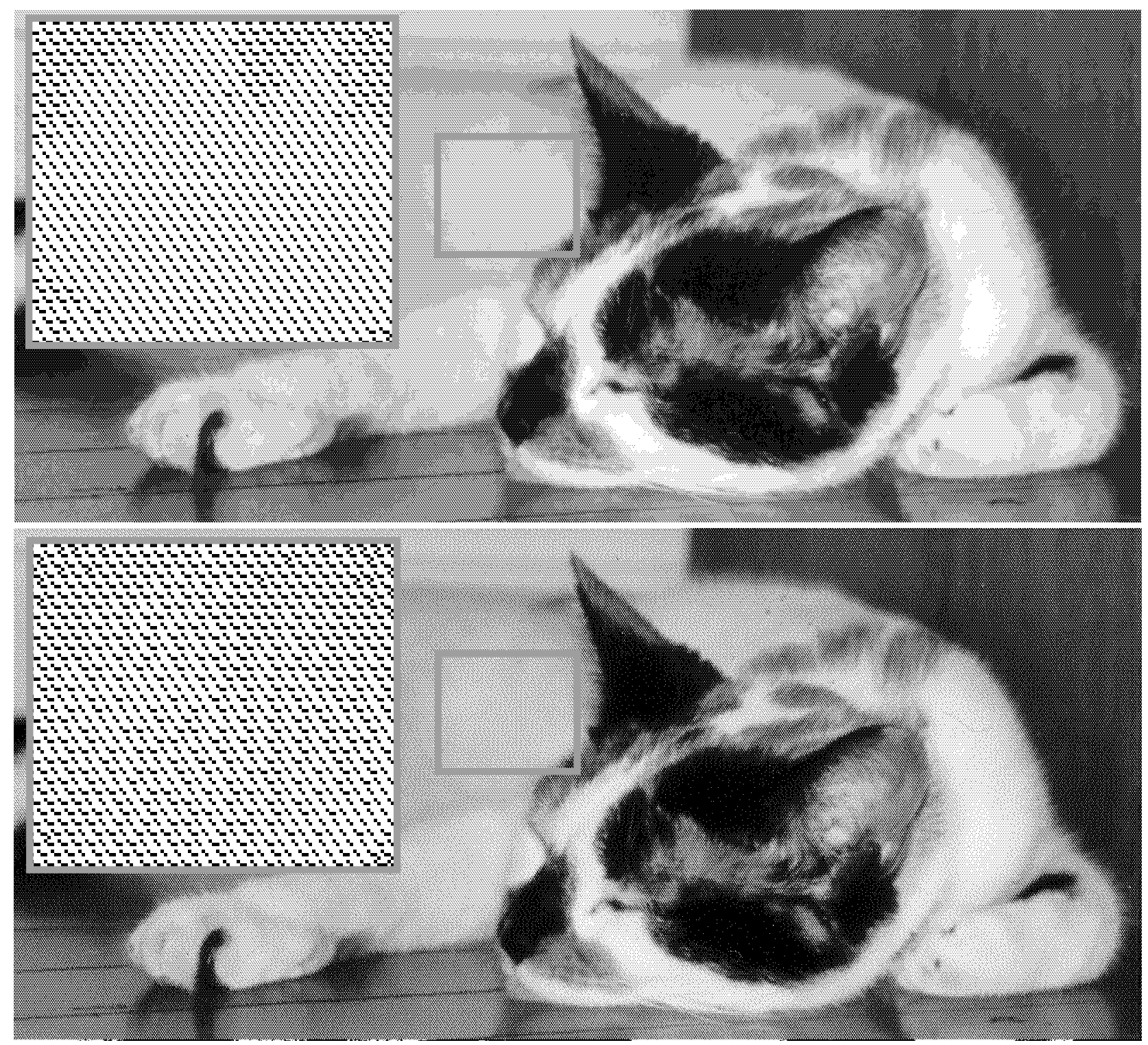

Figure 10. The first result was rendered by simply applying a 12 level clustered-dot matrix over the input image. The second one was computed with the combination of ED_HEXA (.) and the clustered-dot matrix. Note that the micro-structure is the same in both images.

The optimization process using both the simplex downhill search method and the function COST FUNCTION ( . ) converged in less than two minutes on a $1.4 \mathrm{GHz}$ Athlon processor for each key levels. We compared these results with others obtained with a simulated annealing algorithm ${ }^{22}$ and saw that the results were very much similar at every key levels. That observation made us believe that even if the simplex method can theoretically fall into local minimas, in the present case it converges near the global minima. We thus made the decision to keep working with the simplex method, mainly because of its speed.

Concerning the ED_HEXA (.) procedure applied to a perfect hexagonal grid, results are shown in Figure 6 . The reader can see what function ERROR-DIFFUSION ( . ) using Shiau-Fan coefficient set ${ }^{5}$ and a serpentine path gives by opposition to ED_HEXA ( . ) using the optimized coefficient sets. We can see that the latter produces globally less artifacts 
especially around levels 64, 96, 160, 192 and 224.

Finally, results of ED_HEXA (. ) applied over a quasi-hexagonal grid (such as the one in Figure 8) are presented in Figure 9 and 10. Figure 9 shows the difference between ED HEXA ( . ) using Shiau-Fan's coefficient set(SF), our approach and Fan's dot-to-dot algorithm. ${ }^{3}$ We decided to put a grayscale ramp going from $\frac{232}{255}$ to $\frac{255}{255}$ because the clustered-dot matrix with 12 thresholds divides the $0-1$ scale into 12 sections of equal length $\left(\frac{21.3}{256}\right)$ all having a similar configuration. For this reason, the artifacts shown between $\frac{232}{255}$ and $\frac{232}{255}$ are exactly the same between $\frac{211}{255}$ and $\frac{232}{255}, \frac{190}{255}$ and $\frac{211}{255}, \frac{158}{255}$ and $\frac{190}{255}$ and so on down to zero.

Figure 10 shows the striking difference between our approach and a straight 12 levels clustered-dot threshold. Among other things, we can see that our approach minimizes the false contours while preserving the fundamental regular clustered structure of the matrix. The two images were printed in 300 dpi.

\section{CONCLUSION}

In this paper, an optimal error-diffusion technique was introduced. This simple and fast method uniformly distributes pixels over a hexagonal grid. Instead of using the classical error-diffusion algorithm (ERROR-DIFFUSION ( . ) ) we implemented a slightly modified version of that algorithm (ED HEXA ( . ) ). This method takes advantage of 255 coefficient sets, each being optimized in order to minimize artifacts around their corresponding intensity level.

The optimization process used a simplex downhill search method together with the blue noise based cost function COST_FUNCTION (. . . During the optimization process, for a matter of simplicity, we made the decision not to use the hexagonal grid $g_{h}(u, v)$ directly. Instead, an orthogonal grid $g_{o}(x, y)$ was generated and converted to $g_{h}(u, v)$ using a bijection operator $\tau$. By making use of the spectral bijection operator $\mathbf{T}$, the function COST FUNCTION ( . ) calculates the hexagonal grid Radial Fourier Transform (RFT) and measures how close it is to the blue noise profile. After many tests made over a large variety of images, it was found that a configuration with 3 coefficients was a good choice regarding simplicity, speed and better optimization efficiency.

By its conceptual simplicity and speed, our algorithm can be used to work over a variety of printing and visualization devices as illustrated in figure 10. It is thus possible to drive physical hexagonal entities directly, without having to pass through an intermediate orthogonal structure. We have shown that by mixing together clustered-dot matrices and ED_HEXA (. ), the classical problems of dispersed dots can be considerably improved while reducing the false contours.

In the future, we expect to reduce the artifacts around level $\frac{1}{2}$ and $\frac{1}{3}$ and address the question of the uniform areas located around the 12 intensity levels : $21.3,42.6,63.9,85.2, \ldots, 234.6$. We think that this last challenge could be addressed like a multilevel contouring artifact. ${ }^{23,24}$

\section{ACKNOWLEDGMENTS}

The authors would like to thank Pierre Poulin and Justin Bur for their very precious help.

\section{REFERENCES}

1. V. Ostromoukhov, "A simple and efficient error-diffusion algorithm," in SIGGRAPH 2001, Computer Graphics Proceedings, Annual Conference Series, pp. 567-572, 2001.

2. G. Goertzel and G. Thompson, "Digital halftoning on the IBM 4250 printer," IBM Journal of Research and Development 31, pp. 2-15, Jan. 1987.

3. Z. Fan, "Dot-to-dot error diffusion," Journal of Electronic lmaging , Jan.

4. R. Stevenson and G. Arce, "Binary display of hexagonally sampled continuous-tone images," Journal of the Optical Society of America 2 , pp. $1009-1013$, July 1985.

5. J. Shiau and Z. Fan, "A set of easily implementable coefficients in error diffusion with reduced worm artifacts," in SPIE, 2658, pp. 222-225, 1996.

6. P. Li and J. Allebach, "Tone dependent error diffusion," in Proceedings SPIE Conf. Electronic Imaging, pp. 293-301, (San Jose, Ca), 2002.

7. G. Marcu, "An error diffusion algorithm with output position constraints for homogeneous highlights and shadow dot distribution," Journal of Electronic Imaging 9(1), pp. $46-51,2000$.

8. K. Knox, "Error image in error diffusion," in Proceedings SPIE Conf. Electronic Imaging, pp. 9-14, (San Jose, CA), Feb. 1992.

9. R. Eschbach, "Reduction of artifacts in error diffusion by means of input-dependent weights," Journal of Electronic Imaging 2, pp. 352-358, October 1993.

10. R. Ulichney, Digital Halftoning, MIT Press, Cambridge, MA, USA, 1987.

11. R. Floyd and L. Steinberg, "An adaptive algorithm for spatial gray scale," in SID 75, Int. Symp. Dig. Tech. Papers, p. $36,1975$.

12. R. Ulichney, "Dithering with blue noise," Proceedings of the IEEE 76, pp. 56-79, 1988.

13. K. Knox, "Evolution of error diffusion," Electronic Imaging 8(4), pp. 422-429, 1999.

14. F.P. Preparata and M.I. Shamos, Computational Geometry : An Introduction, Springer-Verlag, 1985.

15. R. Ulichney, “A review of halftoning techniques," in Proceedings SPIE Conf., Proc. SPIE 3963, pp. 378-391, 2000. 
16. W. V. et al., Numerical Recipes: Example Book (C), Cambridge University Press, 1998.

17. P. Jodoin and V. Ostromoukhov, "Error-diffusion with blue-noise properties for midtones," in Proceedings SPIE Conf. Electronic Imaging, pp. 293-301, (San Jose, Ca), 2002.

18. J. Gaskill, Linear Systems, Fourier Transforms and Optics, John Willey \& Sons, New York, 1978.

19. K. Knox, "Introduction to digital halftones," in IS\&T's 47th Annual Conference, ICPS'94: The Physics and Chemistry of Imaging Systems, 2, pp. 456-459, May 1994.

20. K.T.Knox, "Evolution of error diffusion," Journal of Electrical Imaging 8(4), pp. 422 - 429, 1999.

21. R. Eschbach, "Pixel-based error-diffusion algorithm for producing clustered halftone dots," Journal of Electronic Imaging 3, pp. 198-202, April 1994.

22. L. Ingber, "Simulated annealing: Practice versus theory," Mathematical and Computer Modeling 18, pp. 29-57, 1993,http://www.ingber.com/.

23. G. Lin and J. Allebach, "Multilevel screen design using direct binary search," in Proceedings SPIE Conf. Electronic Imaging, pp. 264-277, (San Jose, CA), Feb. 2002.

24. K. S. Q. Yu, K. Parker and R. Miller, "Improved digital multitoning with overmodulation scheme," in Proceedings SPIE Conf. Electronic Imaging, pp. 264-277, (San Jose, CA), Feb. 1998.

Table 6. List of the coefficient sets optimized by our method. The key-levels are those highlighted on the left.

d10. d-11. d01.

0: $6691 \quad 0 \quad 3309$

1: 6691 0 3309

2: $\begin{array}{llll}6576 \quad 316 \quad 3108\end{array}$

3: $6462 \quad 629 \quad 2909$

$\begin{array}{llll}4 & 6348 & 940 & 2711\end{array}$

5: 623612482516

6: $6124 \quad 15542322$

7: $6014 \quad 18572129$

8: $5904 \quad 2157 \quad 1938$

9: $5795 \quad 24561749$

10: 568827511561

11: 558130441375

12: 547433351190

13: 536936241007

14: $52653910 \quad 825$

15: 51614194645

$\begin{array}{llll}\text { 15: } 5161 & 4194 & 645 \\ 162 & 4237 & 1081\end{array}$

16: $46824237 \quad 1081$

17: $4303 \quad 4272 \quad 1425$

18: $39974300 \quad 1704$

19: $37434323 \quad 1934$

20: $3530 \quad 4342 \quad 2128$

21: $39004165 \quad 1935$

22: $45163871 \quad 1613$

23: 437537221904

24: 421435512236

25: 402733542619

26: 400037792221

26: 400037792221

$\begin{array}{llll}27: & 3972 & 4224 & 1804\end{array}$

29. 39125177911

29: 39125177911

30: $38795690 \quad 43$

31:3785 5701514 d10. d-11. d01.

32: 36935712595

33: 36035722675

34: 35145733753

35: 35095694798

36: 35045655841

37: $34995618 \quad 883$

38: 34945518883

38. 34895581925

39. $34895545 \quad 965$

40: 348555101005

41: 348054761044

42: 347654421082

43: 347154091120

44: 339951391462

45: 333348911776

46: 327246642064

47: $32164454 \quad 2330$

48: 316442602576

49: $31164080 \quad 2804$

51: 302937563215

52: 299036103400

53: 295434733574

54: 291933443737

55: 288732233890

56: 285631094034

57: 282730024171

58: $2800 \quad 29004300$

58: $5800 \quad 2900 \quad 4300$

59: $27742804 \quad 4422$

61: $34603942 \quad 2598$

62: 375744351808

63: 402948861086 d10. d-11. d01.

64: $4278 \quad 5300 \quad 42$

65: $42495324 \quad 427$

66: $42205347 \quad 432$

67: $4192 \quad 5371 \quad 437$

68: $41635395 \quad 442$

69: 41345418447

70: $4106 \quad 5442 \quad 452$

71: $40775465 \quad 457$

73: $4020 \quad 5512467$

74: 39925536472

75: 39645559477

76: 39365582482

77: 39075605487

78: 38795628492

79: 38515652497

80: 38235675

81: 3795

82. 37685721512

83: 37405744517

84: 3712576752

85: 36845789526

86: 37435747510

87: 38025705493

88: 38605663477

89: 39185622461

90: $3975 \quad 5580 \quad 44$

91: $4032 \quad 5539 \quad 428$

92. 40895498412

93: 41465458396

94: 4202541738

95: 42585377365 d10. d-11. d01

96: 43135337349

97: $43695298 \quad 334$

98: 44245258318

99: 44785219303

100: 45325180288

101: 45865141273

102: 46405103258

103: 46935064243

104: 47465026228

105: 47994988213

106: 48514950198

107: $4904 \quad 4913 \quad 183$

108: 49554876169

109: 50074839154

110: $5058 \quad 4802 \quad 140$

111: $51094765 \quad 126$

112. $51604729 \quad 111$

112: $51604729 \quad 11$

113: $52104693 \quad 97$

114: $52604657 \quad 83$

15: 5310462169

116: 5360458555

117: 5409455041

118: 5458451427

119: $5507 \quad 4479 \quad 14$

120: 555644440

121: 5506440391

122. 54484356196

122: 53804356196

123: 53804299321

125. 52004150650

125: 52004150650

126: $50774048 \quad 875$

127: $49203918 \quad 1162$ 